\title{
From synchronous to one-time delayed dynamics in coupled maps
}

\author{
Celia Anteneodo, ${ }^{1, *}$ Juan Carlos González-Avella, ${ }^{1,2, \dagger}$ and Raúl O. Vallejos ${ }^{3, \sharp}$ \\ ${ }^{1}$ Department of Physics, PUC-Rio, Caixa Postal 38097, 22451-900, Rio de Janeiro, Brazil \\ ${ }^{2}$ Instituto de Física Interdisciplinar y Sistemas Complejos IFISC (CSIC-UIB), Campus Universitat de les Illes Balears, \\ 07122 Palma de Mallorca, Spain \\ ${ }^{3}$ Centro Brasileiro de Pesquisas Físicas (CBPF), Rua Dr. Xavier Sigaud 150, 22290-180 Rio de Janeiro, Brazil
}

(Received 19 April 2017; published 16 June 2017)

\begin{abstract}
We study the completely synchronized states (CSSs) of a system of coupled logistic maps as a function of three parameters: interaction strength $(\varepsilon)$, range of the interaction $(\alpha)$, that can vary from first neighbors to global coupling, and a parameter $(\beta)$ that allows one to scan continuously from nondelayed to one-time delayed dynamics. In the $\alpha-\varepsilon$ plane we identify periodic orbits, limit cycles, and chaotic trajectories, and describe how these structures change with delay. These features can be explained by studying the bifurcation diagrams of a two-dimensional nondelayed map. This allows us to understand the effects of one-time delays on CSSs, e.g., regularization of chaotic orbits and synchronization of short-range coupled maps, observed when the dynamics is moderately delayed. Finally, we substitute the logistic map with cubic and logarithmic maps, in order to test the robustness of our findings.
\end{abstract}

DOI: 10.1103/PhysRevE.95.062213

\section{INTRODUCTION}

Coupled-map lattices are paradigmatic models for the study of complex collective behavior such as synchronization [1-4]. This collective phenomenon presents scientific and technological interest for such diverse areas as Josephson junction arrays, multimode lasers, vortex dynamics, even evolutionary biology [1], and also constitutes good prototypes to investigate the control of chaos.

Asymptotic collective states can be highly influenced by asynchronicities, which are present in real systems [5-9]. For instance, asynchronous updating may open windows in parameter space where synchronization becomes allowed [6] and induce regularity in coupled systems, in contrast to a synchronous updating $[5,10,11]$. Similarly, the introduction of time delays, to account for finite propagation times in information transmission among units [12-15], noticeably impacts the collective patterns, e.g., although synchronization is still possible, chaos may be suppressed [12].

Within the spatial domain, a realistic ingredient that has an important impact on the determination of the emergent patterns in extended systems is the coupling range [13,16-23]. Insofar as the range of the interactions can affect the propagation of information, it is important to explore its interplay with the updating scheme.

In this article we propose a scheme of coupled maps that integrates these two characteristics, allowing one to explore its interplay. Namely, the coupling depends both on the distance among units, covering from nearest-neighbor to global interactions, as well as on a delay protocol that scans continuously from the fully synchronous to the fully one-time delayed dynamics. In particular, we monitor the emergence and breakdown of phase space structures, such as fixed points, periodic orbits, limit cycles, etc., in completely synchronized

\footnotetext{
*celia.fis@puc-rio.br

†avellaj@gmail.com

${ }^{\ddagger}$ vallejos@cbpf.br
}

states (CSSs), when the contribution of delays and the range of the interactions change between extreme cases.

\section{THE MODEL}

We consider a linear chain of $N$ maps with periodic boundary conditions. The maps are coupled according to the diffusive scheme and obey the following dynamical equations,

$$
x_{t+1}^{i}=(1-\varepsilon) f\left(x_{t}^{i}\right)+\varepsilon \sum_{r=1}^{N^{\prime}} A(r)\left[f\left(\hat{x}_{t}^{i-r}\right)+f\left(\hat{x}_{t}^{i+r}\right)\right],
$$

for $i=1, \ldots, N$. Here, $x_{t}^{i}$ describes the state of map $i$ at discrete time $t$, whose uncoupled dynamics is governed by the chaotic map $f(x)$. Delayed coordinates $\hat{x}_{t}^{j}$ are defined by

$$
\hat{x}_{t}^{j}=\beta x_{t-1}^{j}+(1-\beta) x_{t}^{j} .
$$

Thus, the interpolation parameter $\beta(0 \leqslant \beta \leqslant 1)$ allows one to scan continuously from the synchronous or nondelayed dynamics $(\beta=0)$, to the pure one-time delayed dynamics $(\beta=1)$. Equation (1) describes a fully connected array where elements interact through a coupling which depends on $f(x)$, with intensity $A(r)$, where $r$ is the integer distance between maps on the ring. Finally, the coupling parameter $\varepsilon(0 \leqslant \varepsilon \leqslant 1)$ sets the relative weights of global and local influences. At each time step, the $N$ maps are updated simultaneously.

In numerical examples, we will consider $A(r)=r^{-\alpha} / \eta$, where $\alpha \in[0, \infty)$ determines the range of the interactions, and $\eta(\alpha)=2 \sum_{r=1}^{N^{\prime}} r^{-\alpha}$ is a normalization factor, with $N^{\prime}=(N-$ 1)/2 for odd $N$. This coupling scheme allows one to switch smoothly from global $(\alpha=0)$ to nearest-neighbor $(\alpha \rightarrow \infty)$ interactions.

In numerical simulations we will use, as paradigmatic example, mainly the logistic map $f(x)=4 x(1-x)$. However, we will also show results for logarithmic and cubic maps. In analytical expressions we will keep the generic forms of $A(r)$ and $f(x)$ as far as possible. 


\section{RESULTS}

We performed numerical simulations of the coupled-map lattice defined by Eq. (1), with the forms of $A(r)$ and $f(x)$ described above, starting from random initial conditions (uniform in the interval $[0,1]$ ). We considered different values for the coupling strength $\varepsilon$, the range of the interactions ruled by $\alpha$, and the time delay parameter $\beta$.

We monitored the collective behavior by means of the instantaneous mean field $h_{t}$ defined as [12,22]

$$
h_{t}=\frac{1}{N} \sum_{i=1}^{N} x_{t}^{i} \text {. }
$$

In order to measure the degree of synchronization, we use the time average $\left\langle\sigma_{t}\right\rangle$ of the instantaneous standard deviation of $h_{t}$, namely,

$$
\sigma_{t}=\sqrt{\frac{1}{N} \sum_{i=1}^{N}\left(x_{t}^{i}-h_{t}\right)^{2}} .
$$

When $\sigma \equiv\left\langle\sigma_{t}\right\rangle=0$, it means that the system is completely synchronized, i.e., for all $t$, it holds $x_{t}^{1}=x_{t}^{2}=\cdots x_{t}^{N}=x_{t}^{\star}$, where $x_{t}^{\star}$ can trace a chaotic or a regular trajectory. In the latter case, we measured the period of the short orbits.

Another relevant parameter, which allows one to characterize the orbits of a dynamical system, is the largest Lyapunov exponent $\lambda_{\max }$ [24]. If $\lambda_{\max }$ is positive, the system displays a chaotic behavior, while if it is negative, the dynamics is regular. We computed $\lambda_{\max }$ using the Benettin algorithm [25].

In Fig. 1, we present phase diagrams in the $\varepsilon-\alpha$ plane obtained from numerical simulations, for the local dynamics given by the logistic map $f(x)=4 x(1-x)$, and for different values of $\beta$. For each pair $(\varepsilon, \alpha)$ we calculated $\sigma$ by averaging over 100 time steps, after a transient of $t>10^{3}$. We considered the state as fully synchronized if $\sigma<10^{-3}$. Only the parameter regions where complete synchronization occurs are colored. Shown are regions containing periodic orbits up to period-16. Green regions may contain periodic (period $>16$ ), quasiperiodic, intermittent, or chaotic trajectories (see below).

Figure 1 shows several known features such as the existence of an interval of the coupling strength $\varepsilon$ for the system to synchronize, and that long-range interactions favor synchronization, i.e., the stability of completely synchronized (CS) regions shrinks as $\alpha$ grows. Besides these features, Fig. 1 also exhibits important changes with $\beta$. In particular, there is a qualitative change of the stability regions around $\beta \approx 0.5$.

Let us first look the case $\beta=0.0$ in Fig. 1(a): Here, CSSs are fully chaotic; however, these states only exist above minimum values of (1) interaction strength and (2) interaction range. By increasing the contribution of delayed states $(\beta>0)$, windows of regular behavior are introduced. For instance, in the case $\beta=0.1$, the visible windows of regularity correspond to period-doubling cascades (from right to left) of periods $3 \times 2^{k}$ and $5 \times 2^{k}$, with $k=0,1,2, \ldots$, around $\epsilon \simeq 0.55$ and 0.83 , respectively. In the case $\beta=0.2$, shown in Fig. 1(c), we neatly notice a period-doubling cascade from the right to the left of periods $2^{k}$, with $k=1,2, \ldots$ leading to chaos below $\varepsilon \approx 0.67$. This cascade is shifted to the left as $\beta$ increases.
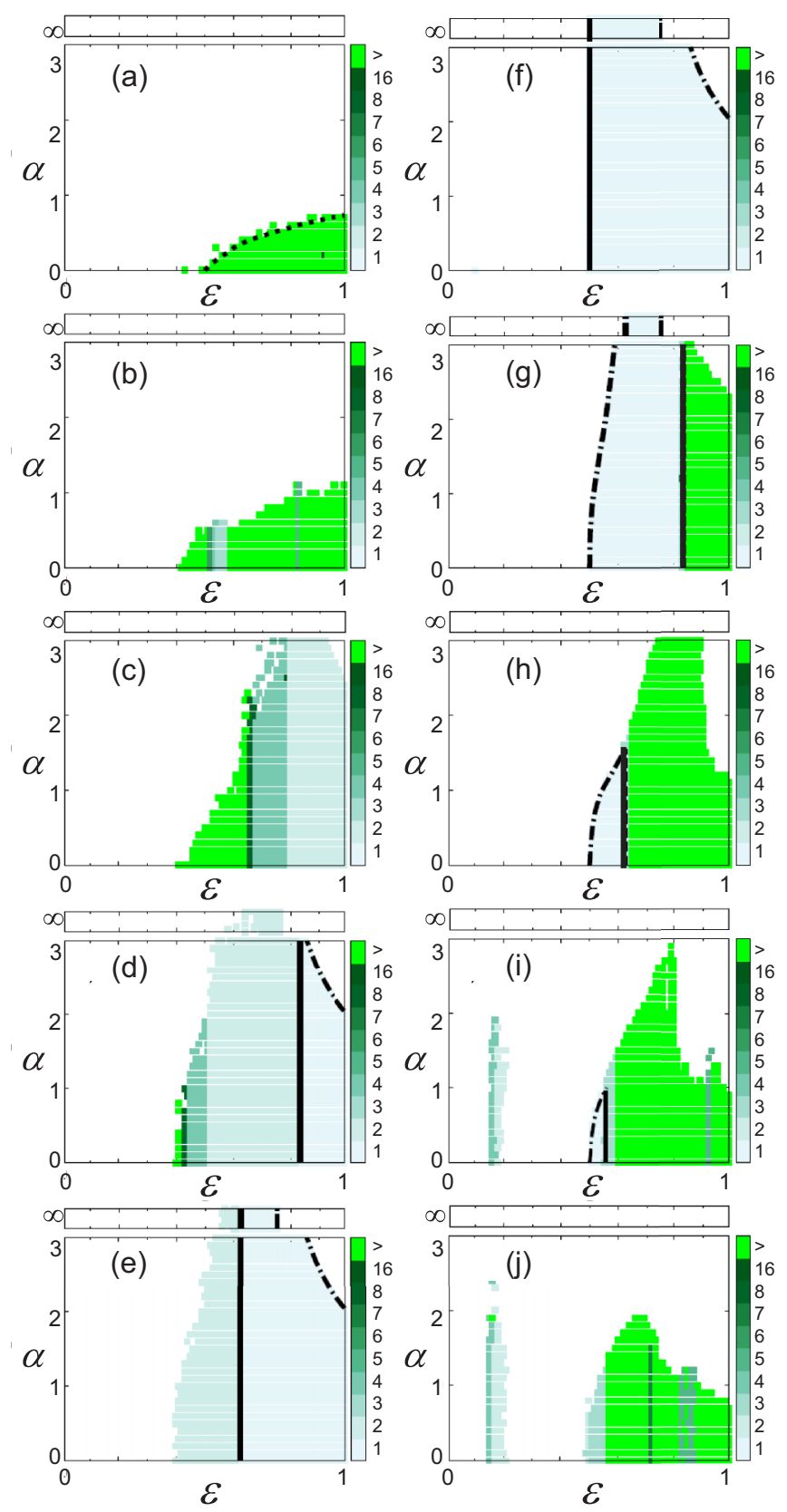

FIG. 1. Phase diagrams in the $\varepsilon-\alpha$ plane for (a) $\beta=0.0$, (b) 0.1 , (c) 0.2 , (d) 0.3, (e) 0.4, (f) 0.5, (g) 0.6, (h) 0.8, (i) 0.9, and (j) 1.0 . Only regions where coupled maps fully synchronize are colored. The color scale indicates the period of the synchronized orbits. The local dynamics corresponds to the logistic map $f(x)=4 x(1-x)$. The black lines indicate the frontiers of the regions of period-1 orbits: Vertical solid lines are given by the longitudinal stability condition (12) and point-dashed lines by the transverse stability condition (13). The dotted line in the first panel is given by Eq. (19). In all cases, the array size is $N=201$. We chose initial conditions $x_{0}^{i}$ and $x_{1}^{i}$ randomly in $[0,1]$.

The larger the delay contribution, the shorter is the range of the interactions that gives rise to CSSs. Moreover, when $\beta \in$ $(\approx 0.3, \approx 0.6)$, i.e., moderate delay contribution, even nearestneighbor couplings allow complete synchronization of regular trajectories. When the contribution of delays is large enough, 
the structure of the bifurcations becomes more complex, and this will be discussed in Sec. IV.

These qualitative changes, that for the logistic map occur around $\beta \approx 0.5$, can be understood heuristically as follows. When complete synchronization occurs, all maps share the same state $x_{t}^{\star}$ at each time step, then, substituting $x_{t}^{i}$ by $x_{t}^{\star}$ into Eq. (1), the summation becomes $\varepsilon f\left(\hat{x}_{t}^{\star}\right)$. Thus, the dependence on $\alpha$ and $N$ embodied in $\eta$ cancels out. Finally, using the definition of $\hat{x}_{t}^{\star}$ given by Eq. (2), Eq. (1) becomes

$$
x_{t+1}^{\star}=(1-\varepsilon) f\left(x_{t}^{\star}\right)+\varepsilon f\left(\beta x_{t-1}^{\star}+\{1-\beta\} x_{t}^{\star}\right) .
$$

The stability of a given trajectory $x_{t}^{\star}$ depends only on $\varepsilon$ and $\beta$. However, when thought of as a CSS of the coupled-map lattice, its stability is also governed by $\alpha$ and $N$, while parameters $\varepsilon$ and $\beta$ only determine the longitudinal stability of CSSs.

For weakly delayed dynamics, the map (5) resembles the logistic map and its bifurcation diagram is logisticlike. However, for delay-dominated dynamics $(\beta>0.5$ in this case), the dynamics of CSSs becomes really two dimensional and the family of bifurcations expands. We return to this issue in Sec. IV.

Now, let us analyze the stability of CSSs, starting by periodic motion.

\section{A. Stability of CS fixed points}

If the system synchronizes at a fixed point $X^{0}$, then for each element $i$, one has $x_{t}^{i}=x_{t-1}^{i}=x_{t}^{\star}=X^{0}$, hence $\hat{x}_{t}^{i}=\beta x_{t-1}^{i}+$ $(1-\beta) x_{t-0}^{i}=X^{0}$. Since, from Eq. (1), each equation in the array verifies the single map relation $X^{0}=f\left(X^{0}\right)$, then the fixed points of the array are identical to the fixed points of the uncoupled maps. For instance, in the case $f(x)=4 x(1-x)$, the fixed points are $X^{0}=0$ and $X^{0}=\frac{3}{4}$, which are unstable in the uncoupled map, since in both cases $\left|f^{\prime}\left(X^{0}\right)\right|>1$. However, their stability is expected to change in the array.

In order to study the stability (both longitudinal and transversal) of the fixed points of the coupled system, we consider the linearized form of Eq. (1) around a fixed point $X^{0}$, which reads

$$
\delta x_{t+1}^{i}=(1-\varepsilon) D^{0} \delta x_{t}^{i}+\varepsilon \sum_{r=1}^{N^{\prime}} A(r) D^{0}\left(\delta \hat{x}_{t}^{i-r}+\delta \hat{x}_{t}^{i+r}\right),
$$

where $D^{0} \equiv f^{\prime}\left(X^{0}\right)$ and $\delta \hat{x}_{t}^{j}=\beta \delta x_{t-1}^{j}+(1-\beta) \delta x_{t-0}^{j}$. The system of linear equations (6) can be cast in the form

$$
\delta \tilde{\mathbf{x}}_{t+1}=\mathbf{F}^{\mathbf{0}} \delta \tilde{\mathbf{x}}_{t}
$$

by defining the $2 N$-dimensional tangent vector $\delta \tilde{\mathbf{x}}_{t}=$ $\left(\delta \mathbf{x}_{t}, \delta \mathbf{x}_{t-1}\right)^{t}$, and the $2 N \times 2 N$ (time-independent) matrix $\mathbf{F}^{\mathbf{0}}$ :

$$
\mathbf{F}^{\mathbf{0}}=\left(\begin{array}{c|c}
D^{0}[(1-\varepsilon) \mathbb{1}+(1-\beta) \varepsilon \mathbf{A}] & D^{0} \beta \varepsilon \mathbf{A} \\
\hline \mathbb{1} & \mathbb{Q}
\end{array}\right),
$$

where $\mathbf{A}$ is the $N \times N$ matrix whose elements are $A_{i j}=(1-$ $\left.\delta_{i j}\right) A\left(r_{i j}\right)$ and $r_{i j}$ is the distance between elements $i$ and $j$ on the circle, i.e., $r_{i j}=\min _{k}|i-j+k N|$. The eigenvalues of $\mathbf{A}$ are obtained by Fourier diagonalization [17],

$$
a_{k}=2 \sum_{m=1}^{N^{\prime}} A(m) \cos (2 \pi k m / N),
$$

for $1 \leqslant k \leqslant N$. By setting $\mathbf{F}^{\mathbf{0}}(\mathbf{u}, \mathbf{v})^{t}=\lambda(\mathbf{u}, \mathbf{v})^{t}$, and using Eq. (8), we obtain

$$
\begin{aligned}
D^{0}[(1-\varepsilon)+(1-\beta) \varepsilon \mathbf{A}] \mathbf{u}+D^{0} \beta \varepsilon \mathbf{A} \mathbf{v} & =\lambda \mathbf{u}, \\
\mathbf{u} & =\lambda \mathbf{v} .
\end{aligned}
$$

Simple algebra shows that the eigenvalues $\lambda$ of the block matrix $\mathbf{F}^{\mathbf{0}}$ are related to the eigenvalues $a_{k}(k=1, \ldots, N)$ of $\mathbf{A}$, through the characteristic equations

$$
\lambda^{2}-\lambda D^{0}\left[1-\varepsilon+\varepsilon(1-\beta) a_{k}\right]-D^{0} \beta \varepsilon a_{k}=0,
$$

each $a_{k}$ giving two values of $\lambda$. The conditions $|\lambda|<1$ for all eigenvalue $a_{k}$ determine the region of stability of the fixed point in the parameter space $(\alpha, \beta, \varepsilon)$. The spectrum of $\mathbf{A}$ has been analyzed elsewhere [17]. The largest eigenvalue of $\mathbf{A}$ is $a_{N}=1$ for all $\alpha$. The corresponding eigenvector is proportional to $\mathbf{e}_{N}=(1,1,1, \ldots, 1)$, that is, it belongs to the completely synchronized subspace. Therefore, $\left|\lambda_{N}\right|<1$ is the condition for longitudinal stability (along the CS subspace). The remaining eigenvalues provide the conditions for transverse stability.

For the logistic map, the fixed point $X^{0}=0$ remains longitudinally unstable in the array for any $(\varepsilon, \beta)$. For $X^{0}=\frac{3}{4}$, using $a_{N}=1$ in Eq. (11), and setting $|\lambda|<1$, we obtain the condition

$$
\frac{1}{4}<\beta \varepsilon<\frac{1}{2}
$$

which guarantees longitudinal stability. Therefore, depending on $\varepsilon$ and $\beta$, this fixed point can gain stability. In particular, for $\beta=0$ (no delay), the fixed point $X^{0}=\frac{3}{4}$ cannot be stable for any coupling strength $\varepsilon$, but as $\beta$ grows beyond $\beta=\frac{1}{4}$, there appears an interval of values of $\varepsilon$ for which the fixed point becomes stable. This shows the emergence of the longitudinal stability of the locally unstable fixed point as the delay increases. Concerning transverse stability, we checked numerically that eigenvalues $0<a_{k}<1$ do not add restrictions to the region of stability defined by $a_{N}=1$. Eigenvalues $a_{\min }<a_{k}<0$ set the frontier

$$
\frac{1}{2\left[1-a_{\min }(1-2 \beta)\right]}<\varepsilon<\frac{3}{2\left(1-a_{\min }\right)},
$$

recalling that $a_{\min }=a_{N / 2}$ (for $N$ odd). This restriction depends both on $\alpha$ and $N$ through $a_{\mathrm{min}}$. However, the $N$ dependence is very weak for $N$ large, e.g., $N=100$. These frontiers are represented by lines in Fig. 1 .

Equation (12) shows how the contribution of delays, through $\beta$, directly influences the longitudinal stability of fixed points, which occurs for a moderate range of values of $\beta$. Moreover, Eq. (13) shows how $\beta$ influences the lower bound of transverse stability, turning complete synchronization at the fixed point visible. This condition is relevant only for $\beta>0.5$, because the condition of longitudinal stability $\varepsilon>1 /(4 \beta)$ is more restrictive in such a case.

Complete synchronization at the fixed point $X^{0}=\frac{3}{4}$ can be seen in Fig. 2(a), for $\beta=0.3$, and in Fig. 2(b) for $\beta=0.8$, when $\alpha=0.5$. The intervals of stability as a function of $\varepsilon$ coincide with the analytical calculation, i.e., $\varepsilon>0.833(\beta=$ $0.3)$ and $0.510<\varepsilon<0.625(\beta=0.8)$. 

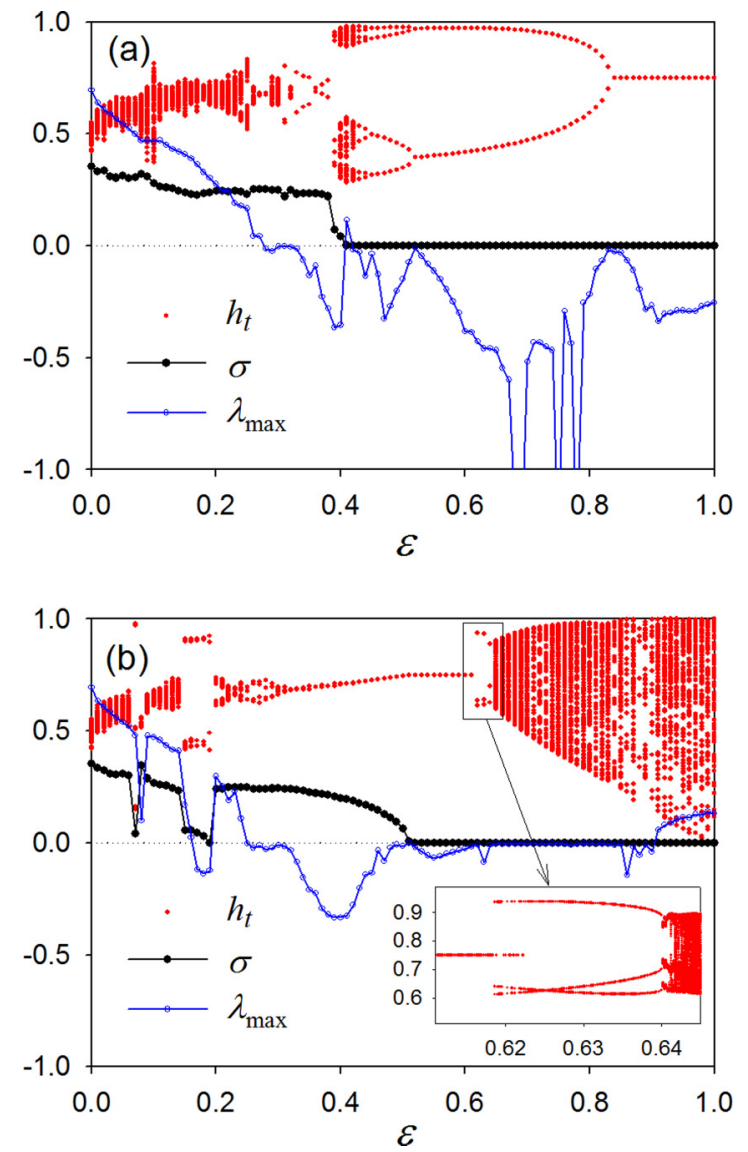

FIG. 2. Order parameter $\sigma$ (black dots), mean field $h_{t}$ (red light dots; for each value of $\varepsilon$ we plot 100 consecutive values after a transient of $t>10^{3}$ ), and largest Lyapunov exponent $\lambda_{\max }$ vs coupling $\varepsilon$, for $\alpha=0.5$, with (a) $\beta=0.3$ and (b) $\beta=0.8$. The local dynamics is given by the logistic map. In all cases the array size is $N=201$. The inset is a zoom of the main frame to show the coexistence of period-1 and period-3 orbits. At the point where two branches of period-3 orbits cross, two of the three values become equal, but remaining period-3. This is possible because the state of the system depends on two previous times.

\section{B. Stability of CS period-2 trajectories}

A period-2 trajectory of the coupled system (with values $X^{1}$ and $X^{2}$ ) must verify

$$
\begin{aligned}
& X^{1}=(1-\varepsilon) f\left(X^{2}\right)+\varepsilon f\left(\beta X^{1}+[1-\beta] X^{2}\right), \\
& X^{2}=(1-\varepsilon) f\left(X^{1}\right)+\varepsilon f\left(\beta X^{2}+[1-\beta] X^{1}\right) .
\end{aligned}
$$

For the logistic map, besides the trivial solutions $\left(X^{1}, X^{2}\right)=$ $(0,0)$ and $\left(\frac{3}{4}, \frac{3}{4}\right)$, there are the (equivalent) nontrivial solutions $\left(X^{1}, X^{2}\right),\left(X^{2}, X^{1}\right)$ with $X^{1} \neq X^{2}$, which are a function of $\varepsilon$ and $\beta$. In order to study the stability of period-2 orbits, we write the evolution of the tangent vectors as

$$
\delta \tilde{\mathbf{x}}_{t+2}=\mathbf{F}^{\mathbf{1}} \mathbf{F}^{2} \delta \tilde{\mathbf{x}}_{t}
$$

where

$$
\mathbf{F}^{\mathbf{k}}=\left(\begin{array}{c|c}
\left.D^{k}(1-\varepsilon) \mathbb{1}+\tilde{D}^{k}(1-\beta) \varepsilon \mathbf{A}\right] & \tilde{D}^{k} \beta \varepsilon \mathbf{A} \\
\hline \mathbb{1} & \mathbb{D}
\end{array}\right),
$$

with $D^{k}=f^{\prime}\left(X^{k}\right)$ and $\tilde{D}^{k}=f^{\prime}\left(\beta X^{j}+[1-\beta] X^{k}\right)$, for $k=$ 1,2 and $j \neq k$. Again, the condition $|\lambda|<1$ for all $a_{k}$ (with $\lambda$ eigenvalue of $\mathbf{F}^{\mathbf{2}} \mathbf{F}^{\mathbf{1}}$ ) provides the region of stability of the period- 2 orbits. This case, however, is not amenable to analytical treatment because the trajectories must be found numerically.

Stable period-2 orbits can be observed in Fig. 2(a) for $\beta=0.3$, within the interval $0.52 \lesssim \varepsilon \lesssim 0.83$. This result is in full agreement with that calculated from the eigenvalues $\lambda\left(a_{k}\right)$ of the matrix $\mathbf{F}^{\mathbf{2}} \mathbf{F}^{\mathbf{1}}$ (details not shown). It is apparent that the period-2 family disappears through an inverse perioddoubling bifurcation, where a fixed point is born. We already calculated analytically where this bifurcation occurs (see the preceding section), namely, $\varepsilon \approx 0.833$, which also agrees with the previous values.

\section{Stability of CS period- $p$ trajectories}

In the coupled system, a period- $p$ trajectory (with values $\left.X^{1}, X^{2}, \ldots, X^{p}\right)$ must verify

$$
\begin{aligned}
X^{1} & =(1-\varepsilon) f\left(X^{p}\right)+\varepsilon f\left(\beta X^{p-1}+[1-\beta] X^{p}\right), \\
X^{2} & =(1-\varepsilon) f\left(X^{1}\right)+\varepsilon f\left(\beta X^{p}+[1-\beta] X^{1}\right), \\
\vdots & =\vdots \\
X^{p} & =(1-\varepsilon) f\left(X^{p-1}\right)+\varepsilon f\left(\beta X^{p-2}+[1-\beta] X^{p-1}\right) .
\end{aligned}
$$

Its stability matrix is given by the product $\mathbf{F}^{\mathbf{p}} \cdots \mathbf{F}^{\mathbf{2}} \mathbf{F}^{\mathbf{1}}$, where we have used the natural extension of the notation of the previous section.

In the particular case $p=3$, a stable family can be observed in Fig. 2(b) in the interval $0.6077 \lesssim \varepsilon \lesssim 0.6401$ (we checked that this interval coincides with the calculation based on the stability matrix). The latter interval overlaps partially with the family of fixed points $(0.510 \lesssim \varepsilon \leqslant 0.625)$, indicating the coexistence of two different families of periodic orbits (bistability).

\section{Stability of an arbitrary CS trajectory}

For calculating the stability of long trajectories (periodic, quasiperiodic, chaotic, etc.), in principle one must resort to purely numerical calculations. Consider an arbitrary orbit of length $L$ generated by the map of Eq. (5), starting from appropriate initial conditions for selecting the desired orbit. $L$ must be large enough for the orbit to fall onto the attractor; a transient of length $M$ may be discarded. The stability matrix of such an orbit reads $\mathbf{F}^{\mathbf{L}} \mathbf{F}^{\mathbf{L}-\mathbf{1}} \cdots \mathbf{F}^{\mathbf{M}+\mathbf{2}} \mathbf{F}^{\mathbf{M}+\mathbf{1}}$. Upon diagonalization, this matrix product decouples into $N$ matrices of $2 \times 2$, each one corresponding to one eigenvalue $a_{k}$ of $\mathbf{A}$. By analyzing the eigenvalues of all $2 \times 2$ matrices we obtain the stability of the trajectory.

Concerning the possibility of analytical calculations, some results can be obtained in the case of chaotic orbits. In the particular case $\beta=0$ (no delay) each map evolves with the uncoupled local chaotic dynamics. All maps are in the same state $X^{t}$ at time $t$ : The full interval $[0,1]$ is a smooth attractor. The one-step stability matrix reads

$$
\mathbf{F}^{t}=[(1-\varepsilon)+\varepsilon \mathbf{A}] f^{\prime}\left(X^{t}\right) .
$$


This leads to the well-known stability condition (see, e.g., Refs. [17,22])

$$
-1 \leqslant e^{\lambda_{u}}\left[1-\varepsilon\left(1-a_{k}\right)\right] \leqslant 1, \quad \text { for all } k<N,
$$

where $\lambda_{u}$ is the Lyapunov exponent of the uncoupled map. The domain of chaotic synchronization defined by the double inequality (19) defines the region in the $\varepsilon$ - $\alpha$ plane below the dashed line in Fig. 1(a) (case $\beta=0$ ). The critical strength $\varepsilon_{c}$ increases with $\alpha$. For instance, for $\alpha=0$ (global coupling, mean field), $\varepsilon_{c}=0.5$. The synchronization interval shrinks as $\alpha$ grows and collapses at $\alpha \simeq 0$.8. Therefore, too short-range interactions are not able to fully synchronize the system $[6,17]$.

For $\beta>0$, from a technical point of view, the stability matrices acquire the $2 \times 2$ block structure shown in Eq. (8) and it is not possible to extract the factor $f^{\prime}\left(X^{t}\right)$. At a more intuitive level, as soon as $\beta$ is non-null, the bifurcation diagram becomes fractal, and it is clear that analytical treatments are extremely difficult: $\beta$ is not a perturbation parameter.

\section{TWO-DIMENSIONAL MAP}

We have shown that in CSSs the dynamics is governed by Eq. (5), which is independent on $\alpha$ and $N$, although these parameters still rule the stability of CSSs. Then, Eq. (5), which in essence is a two-dimensional (2D) map (since it depends on two times), determines the structure of bifurcations of CSSs. In Fig. 3(a) we plot the period of the asymptotic solutions in the $\beta-\varepsilon$ plane (up to period-16); longer-period or nonperiodic trajectories are represented by green pixels. For comparison we have drawn analogous figures for the system of coupled maps at full coupling, i.e., $\alpha=0$ [Fig. 3(b)] and first-neighbor coupling, $\alpha=\infty$ [Fig. 3(c)]. White regions in Figs. 3(b) and 3(c) consist of values of $(\beta, \varepsilon)$ which do not generate stable CSSs for random initial conditions in $[0,1]$. Note that white regions spread as $\alpha$ grows. In particular, the region of period-1 synchronization shrinks as the range of the interaction decreases. For $\alpha=\infty$ only a trianglelike stability region remains, corresponding to low-period orbits (mainly period-1). In order to look into the structure of Fig. 3(a) with greater detail we plotted bifurcation diagrams at $\beta=0.3$ and $\beta=0.8$ for the 2D map. See Figs. 4(a) and 4(b), respectively. Bifurcation diagrams as a function of $\beta$ for fixed $\alpha$ are qualitatively similar to those shown in Fig. 4, since both parameters play a similar role for the 2D map. This feature is evident in Fig. 3(a), by observing the approximate symmetry with respect to the diagonal.

A first look at Fig. 3(b), corresponding to the globally coupled case, shows that most of the synchronized states of the system of coupled maps lie above $\varepsilon=0.4-0.5$. In fact, a minimum coupling is expected for complete synchronization. There is a small island of stability at $\varepsilon \approx 0.2$, and the existence of other small structures is not discarded. However, very small regions are difficult to detect numerically because, presumably, they possess very small basins of attraction, then fine tuning of initial conditions would be required. Consider, for instance, the surprising Fig. 1(f), for $\beta=0.5$. This figure suggests that only period-1 orbits are stable. However, the associated bifurcation diagram for the 2D map (not shown) exhibits an inverse period-doubling cascade for
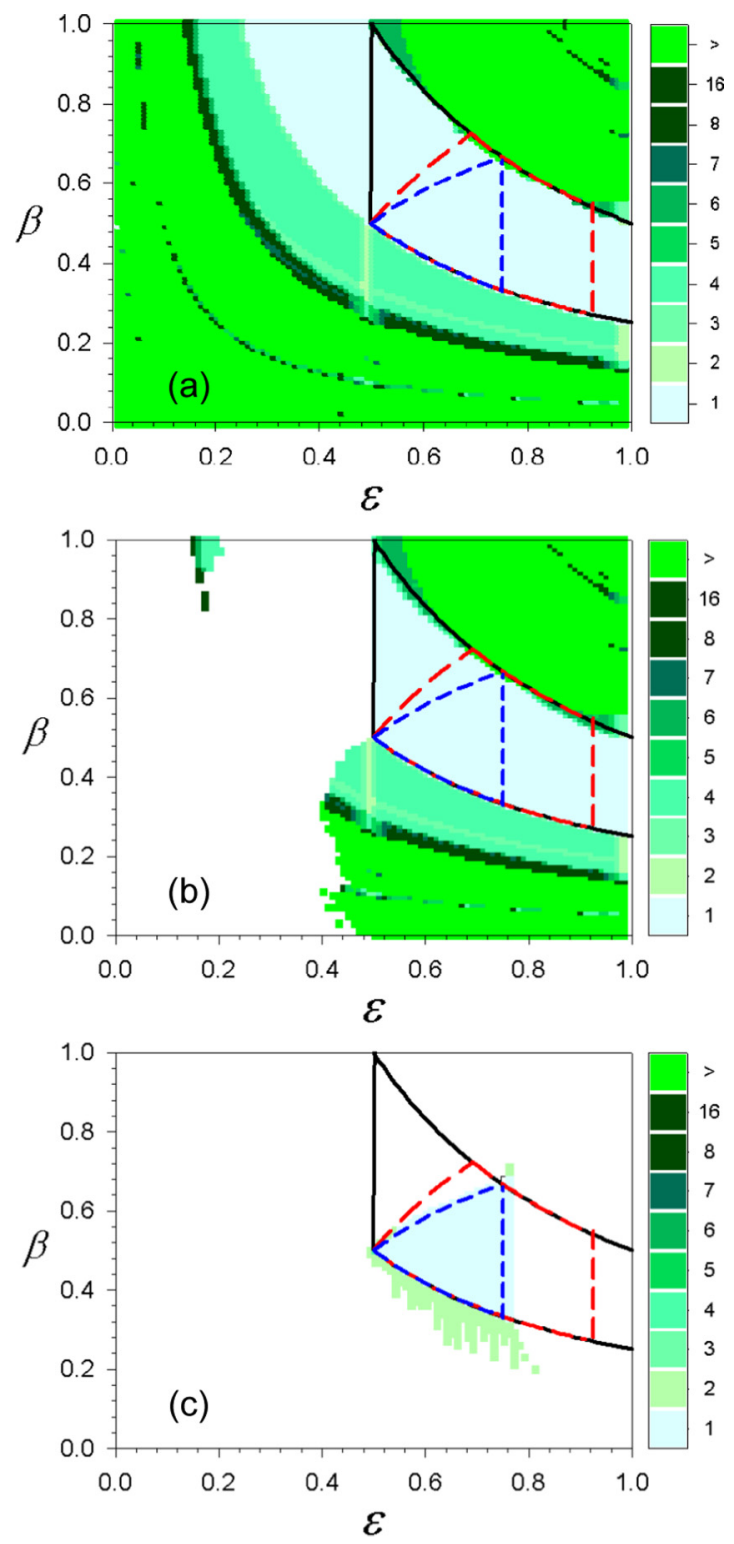

FIG. 3. (a) Asymptotic states of the map of Eq. (5). (b) Stable completely synchronized states of the globally coupled-map system ( $N=201, \alpha=0)$. (c) Same as (b), but for nearest-neighbor couplings $(N=201, \alpha=\infty)$. The local dynamics is that of the logistic map $f(x)=4 x(1-x)$. The region of stability of the fixed point $X^{0}=\frac{3}{4}$ is depicted in light blue. This region is bounded by the arcs of hyperbola given by $\frac{1}{4}<\beta \varepsilon<\frac{1}{2}$ and Eqs. (13) $[\alpha=0$ (solid black); $\alpha=2.4$ (long-dashed red); $\alpha=\infty$ (short-dashed blue)].

$\varepsilon<0.5$ (analogous to those depicted in Fig. 4) and we verified that some of these orbits are indeed stable (e.g., a period-2 orbit at $\varepsilon=0.4$ and $\alpha=0.25)$. So, because of limitations of computing time, Figs. 1 and 3 only exhibit coarse-grained structures. Nonetheless, by complementing these figures with finely detailed bifurcation diagrams we can explain several features of the synchronized states.

For weak delays $(\beta<0.5)$, the bifurcation diagrams are logisticlike, i.e., typical of one-dimensional maps. This can be clearly seen in Fig. 4(a), for $\beta=0.3$. However, for stronger delays the dynamics of synchronized states becomes really 

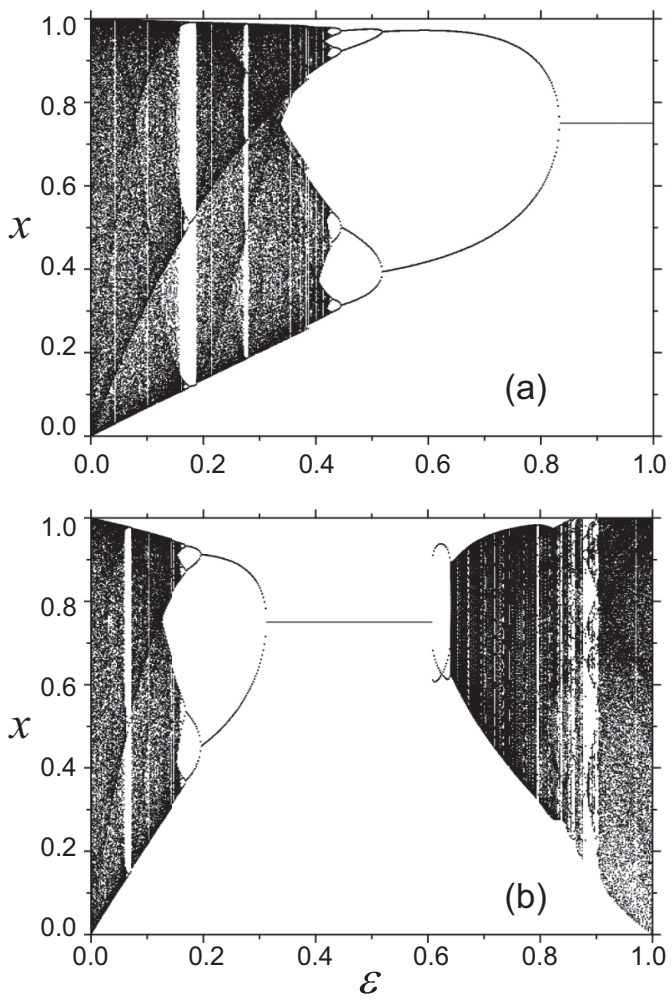

FIG. 4. Bifurcation diagram of the completely synchronized map of Eq. (5) for (a) $\beta=0.3$ and (b) $\beta=0.8$.

two dimensional. As a consequence, e.g., for $\beta=0.8$ (and $\varepsilon>$ 0.5 ), we see in Fig. 4(b) bifurcations which are characteristic of 2D maps, e.g., Neimark-Sacker bifurcations [26]. Notice that the light-green region observed in Fig. 1(h) at $\alpha=0.5$ when $\beta=0.8$, contains diverse structures, as can be seen in Fig. 3(a) and also in Fig. 4(b), where the additional information provided by the largest Lyapunov exponent indicates that there are quasiperiodic, long-periodic, and also chaotic orbits.

\section{OTHER LOCAL DYNAMICS}

In order to test the robustness of our findings, we substituted the logistic map in the local dynamics by either cubic or logarithmic maps.

(i) Cubic map [27,28]:

$$
x \rightarrow f(x)=-x^{3}+a x+b,
$$

with $a=1.5$ and $b=-1$. Initial conditions were taken random in $[-1,1]$. By solving $x=f(x)$, we find the fixed point $X^{0} \approx-1.16537$, with $D^{0} \approx-2.5742$.

(ii) Logarithmic map [29]:

$$
x \rightarrow f(x)=c+\ln |x|,
$$

with $c=0.0$. In this case the initial conditions for each map were random in $[-10,10]$. This map has the fixed point $X^{0} \approx$ -0.567143 , with $D^{0} \equiv f^{\prime}\left(X^{0}\right)=1 / X^{0} \approx-1.763223$. The longitudinal stability condition is $0.216 \lesssim \beta \varepsilon \lesssim 0.567$.

The cubic map is not unimodal and the logarithmic map is neither unimodal nor bounded [29]. The cubic map has
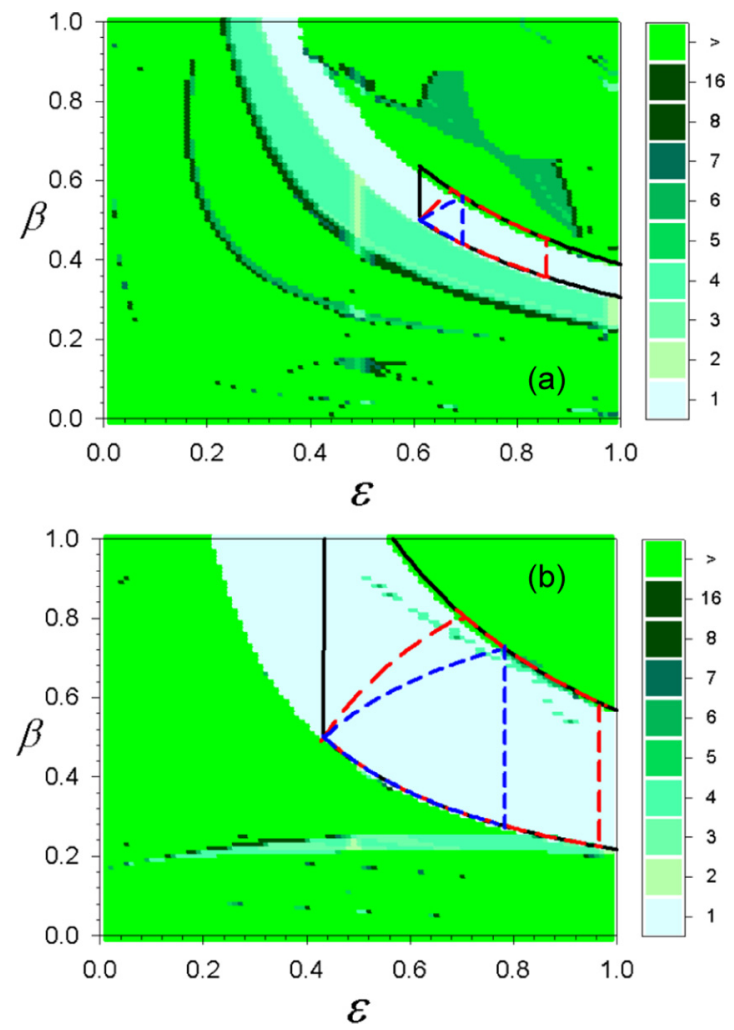

FIG. 5. Phase diagram in the parameter plane $(\varepsilon, \alpha)$ for the (a) cubic and (b) logarithmic delayed 2D maps [cf. Fig. 3(a)]. The color scale shows the period of the orbits in the 2D maps, starting from random initial values in $[-1,1]$ and $[-10,10]$ for the cubic and logarithmic maps, respectively. The lines, given by Eqs. (22) and (23), delimit the region of period-1 complete synchronization.

bifurcation diagrams similar to the logistic one, both as a function of $a$ and $b$. In the logarithmic map bifurcation diagram, as a function of $c$, there is a single fixed point that loses stability for $c \in[-1,1]$ and chaos emerges, without windows of regularity. We built phase diagrams for these maps, shown in Fig. 5, where the frontiers for the fixed point stability in CS states are highlighted.

The longitudinal stability condition, that generalizes Eq. (12) for the logistic map, is

$$
\frac{1+D^{0}}{2 D^{0}}<\beta \varepsilon<-\frac{1}{D^{0}} .
$$

These inequalities define the band of CS period-1 orbits observed in Fig. 5.

The transversal condition generalizing Eq. (13) is given by

$$
\frac{1+D^{0}}{2 D^{0}\left[1-a_{\min }(1-2 \beta)\right]}<\varepsilon<\frac{D^{0}-1}{D^{0}\left(1-a_{\min }\right)} .
$$

This condition depends on the range of the interactions, which restricts the band of period-1 orbits as the range decreases (see Figs. 3 and 5).

The effect of the delay in coupled cubic maps is qualitatively similar to that found for the logistic maps. In fact, the phase diagram of the cubic map, depicted in Fig. 5(a), is a deformation of Fig. 3(a). In both cases the band of CS period-1 orbits separates two regions with either one- or 

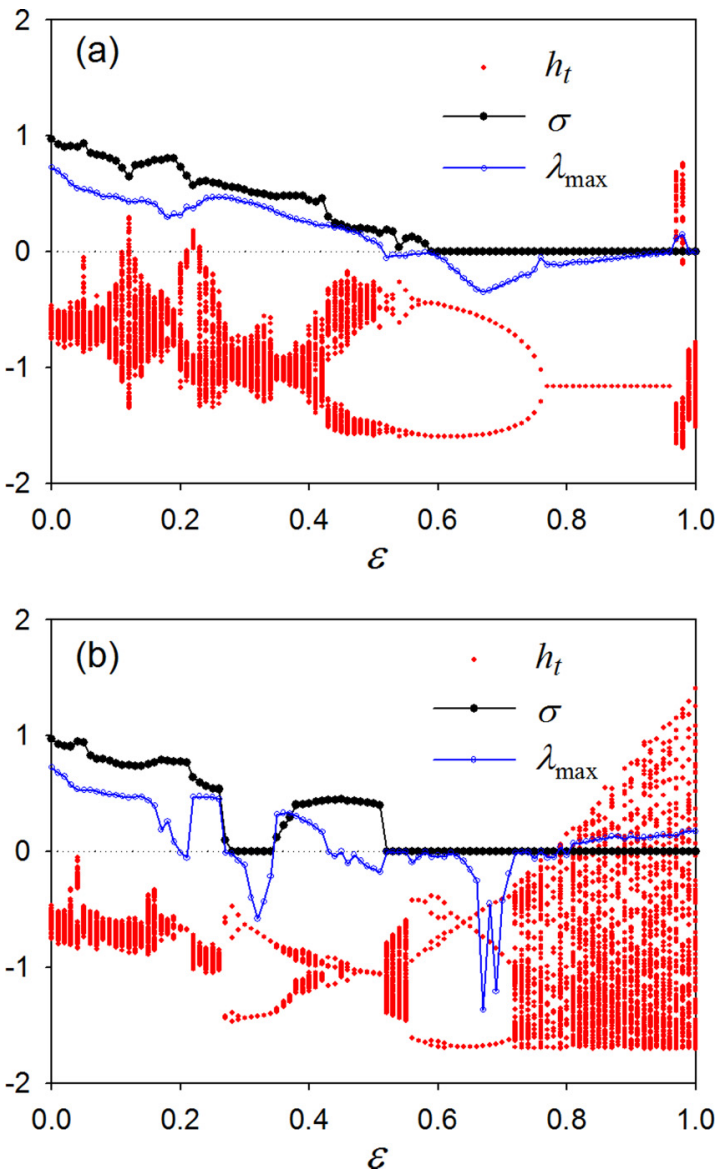

FIG. 6. Cubic maps. Order parameter $\sigma$ (black dots) and mean field $h_{t}$ (red light dots) vs coupling $\varepsilon$, for (a) $\beta=0.4$ and (b) $\beta=0.8$. In all cases the array size is $N=201$ and $\alpha=0.5$.

two-dimensional behavior, as one can see in the bifurcation diagrams in Fig. 6. Incidentally, the structures which appear in Fig. 5(a) remind us the shrimplike structures found in the parameter space of the Hénon map [30,31]. However, in order to certify the existence of such structures, we should select very small regions with a much finer discretization, a study that, although interesting, is beyond our present scope.

In the logarithmic case [Fig. 5(b)] there is also a band of period- 1 orbits, and in the region of small $\beta \varepsilon$, complete synchronization of periodic windows with periods larger than 1 appear (unstable in the uncoupled map), as for the logistic and cubic dynamics. The thin colored region within the period-1 band contains orbits of higher period. For instance, we checked that for $\beta=0.8$ and $\varepsilon \approx 0.65$ there are period-3 orbits coexisting with period- 1 ones-a case of bistability also observed in the logistic system. However, for large $\beta \varepsilon$, the behavior typical of bidimensional maps, with structures such as limit cycles, does not emerge. Bifurcation diagrams are illustrated in Fig. 7, for two different values of $\beta$.

\section{FINAL REMARKS}

We selected a coupled system that scans continuously from synchronized to one-time delayed dynamics. We used the logistic map local dynamics as a paradigm, but general
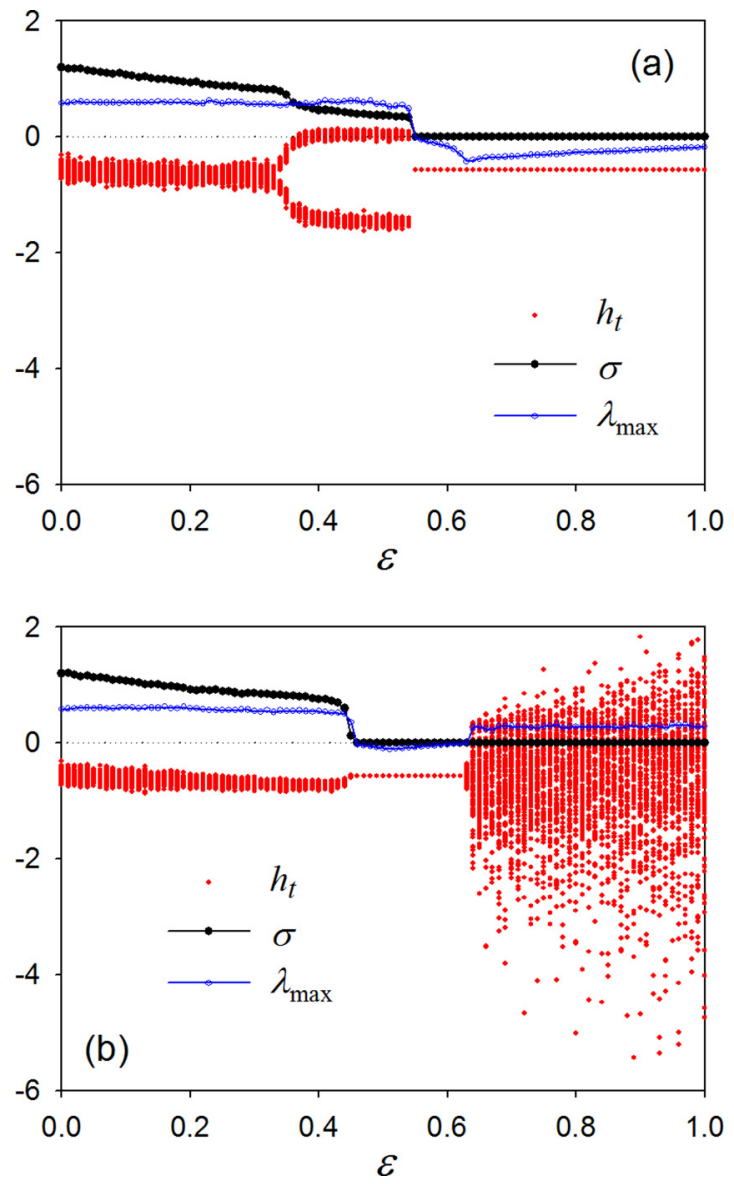

FIG. 7. Logarithmic maps. Order parameter $\sigma$ (black dots) and mean field $h_{t}$ (red light dots) vs coupling $\varepsilon$, for (a) $\beta=0.4$ and (b) $\beta=0.9$. In all cases the array size is $N=201$ and $\alpha=0.5$.

analytical expressions were obtained and other maps were also simulated.

We exhibited the phenomenology that appears in the route of increasing contribution of the delays (increasing $\beta$ ), focusing on complete synchronization. Basically, we distinguish two scenarios: one where delays are not dominant (small enough $\beta$, namely, $\beta<0.5$ in the case of the logistic map), in which case the bifurcation diagram of the coupled maps is qualitatively similar to that for the local dynamics, although deformed. That is, for a small contribution of the delays, the dynamics of completely synchronized states remains essentially one dimensional. The other scenario appears when the dynamics is delay dominated (large enough $\beta$, i.e., $\beta>0.5$ for the logistic map), where a scenario typical of 2D maps emerges. Noticeably, the transition between both kinds of behavior occurs through a fixed point synchronization.

Although in many cases delays regularize the dynamics, we clearly see that they can also have the opposite effect, depending on the coupling parameters. That is, for a small contribution of delays, by increasing $\beta$, chaos (together with the full logisticlike bifurcation diagram) shrinks towards $\varepsilon=$ 0 (see Fig. 4). But for a large contribution of delays, when increasing $\beta$, chaos can originate from the breakdown of limit cycles within the $2 \mathrm{D}$ scenario. The same portrait was observed for the cubic map. 
Despite 2D structures not being observed in the case of local logarithmic dynamics, also here chaos is broken for weak delays and created by strong ones.

We have also shown the interplay of the delays and the range of the interactions. Long-range interactions generically aid complete synchronization, which will not be possible for short-range interactions, in non-delayed dynamics. However, a moderate contribution of delays allows complete synchronization even for nearest neighbors, as displayed, for instance, in Fig. 3.

\section{ACKNOWLEDGMENTS}

We acknowledge Brazilian agencies CNPq and FAPERJ for financial support.
[1] A. Pikovsky, M. Rosenblum, and J. Kurths, Synchronization: A Universal Concept in Nonlinear Sciences, The Cambridge Nonlinear Science Series (Cambridge University Press, Cambridge, UK, 2001).

[2] J. Crutchfield and K. Kaneko, Theory and Applications of Coupled Map Lattices (World Scientific, Singapore, 1987).

[3] S. C. Manrubia, A. S. Mikhailov, and D. Zanette, Emergence of Dynamical Order. Synchronization Phenomena in Complex Systems (World Scientific, Singapore, 2004).

[4] Y. Kuramoto, Chemical Oscillations, Waves, and Turbulence (Springer, Berlin, 1984).

[5] M. D. Shrimali, S. Sinha, and K. Aihara, Phys. Rev. E 76, 046212 (2007).

[6] M. Mehta and S. Sinha, Chaos 10, 350 (2000).

[7] E. D. Lumer and G. Nicolis, Physica D (Amsterdam) 71, 440 (1994).

[8] N. Crokidakis, V. H. Blanco, and C. Anteneodo, Phys. Rev. E 89, 013310 (2014).

[9] N. Crokidakis and C. Anteneodo, Phys. Rev. E 86, 061127 (2012).

[10] H. Atmanspacher, T. Filk, and H. Scheingraber, Eur. Phys. J. B 44, 229 (2005).

[11] H. Atmanspacher and H. Scheingraber, Int. J. Bifurcation Chaos Appl. Sci. Eng. 15, 1665 (2005).

[12] C. Masoller and A. C. Martí, Phys. Rev. Lett. 94, 134102 (2005).

[13] M. Ponce, C. Masoller, and A. C. Martí, Eur. Phys. J. B 67, 83 (2009).

[14] A. C. Martí, M. Ponce, and C. Masoller, Phys. Rev. E 72, 066217 (2005).

[15] Philos. Trans. R. Soc. A 368 (1911), special issue on delayed complex systems, edited by W. Just, A. Pelster, M. Schanz, and E. Schöll.
[16] Y. Kuramoto and H. Nakao, Physica D (Amsterdam) 103, 294 (1997).

[17] C. Anteneodo, A. Batista, and R. Viana, Phys. Lett. A 326, 227 (2004).

[18] C. Anteneodo, A. Batista, and R. Viana, Physica D (Amsterdam) 223, 270 (2006).

[19] P. G. Lind, J. Corte-Real, and J. A. C. Gallas, Phys. Rev. E 69, 026209 (2004).

[20] C. Anteneodo and C. Tsallis, Phys. Rev. Lett. 80, 5313 (1998).

[21] F. Tamarit and C. Anteneodo, Phys. Rev. Lett. 84, 208 (2000).

[22] P. G. Lind, J. A. C. Gallas, and H. J. Herrmann, Phys. Rev. E 70, 056207 (2004).

[23] J. C. González-Avella and C. Anteneodo, Phys. Rev. E 93, 052230 (2016).

[24] J. P. Eckmann and D. Ruelle, Rev. Mod. Phys. 57, 617 (1985).

[25] G. Benettin and J.-M. Strelcyn, Phys. Rev. A 17, 773 (1978).

[26] J. M. T. Thompson and H. B. Stewart, Nonlinear Dynamics and Chaos, 2nd ed. (Wiley, New York, 2002).

[27] J. A. de Oliveira, E. R. Papesso, and E. D. Leonel, Entropy 15, 4310 (2013).

[28] P. G. Lind, S. Titz, T. Kuhlbrodt, J. A. M. Corte-Real, J. Kurths, J. A. C. Gallas, and U. Feudel, Int. J. Bifurcation Chaos 14, 999 (2004).

[29] M. G. Cosenza and J. González, Prog. Theor. Phys. 100, 21 (1998).

[30] J. A. C. Gallas, Phys. Rev. Lett. 70, 2714 (1993).

[31] A. Celestino, C. Manchein, H. A. Albuquerque, and M. W. Beims, Phys. Rev. Lett. 106, 234101 (2011). 